Inventory Nursing Home version (NPI-NH) in 2/5. Network analysis was used to evaluate connectedness of psychopathologic features and a qualitative synthesis distilled recurrent psychopathologic features. Finally, each time point was compared with operationalised diagnoses using an automated classifier and plotted with corresponding symptom complexes over time.

Results All had psychiatric features at onset and were seen first by general practitioners or emergency departments. All received an incorrect initial diagnosis (1 neurological, 4 primary psychiatric). Two patients were referred to mental health services and detained while three were admitted to a general hospital. Psychiatric diagnoses spanned psychotic, mood, and stress categories. None had a personal or family history of serious mental illness or substance misuse. Despite the atypicality all were ascribed to non-specific psycho-social aetiologies. Autoimmune encephalitis was then first suspected between 4-28 days from onset (median $=21$ days) because of the psychopathology $(n=2)$ or development of clear-cut seizures or movement disorder $(n=3) .10$ consistently reported features were identified: sleep disturbance, nightmares, mixed unstable mood, perplexity, incoherent repetitive speech, musical \pm visual hallucinosis, catatonic facies, possession-like/ drugged, dissociative-disorganised, and regressed behaviour.

The symptom complex peaked rapidly (within 3 weeks). The peak burden was large and crossed multiple psychopathologic domains. Overall the syndrome is poorly-described by any single primary disorder; mixtures of mixed mood-psychotic-catatonic disorders performed best. Furthermore, it showed clear qualitative and hence diagnostic shifts between onset, peak, and resolution.

Conclusions The psychopathology of NMDAR-antibody encephalitis is complex and dynamic, likely contributing to diagnostic difficulties. However, it appears stereotyped between individuals, hence sensitive features can be derived. Inconsistency with psychosis and/or mood disorder constructs and better approximation with 'mixtures of mixtures' suggests specificity is possible but similar studies with primary disorder comparators are needed. As the disease can only be ruled out with cerebrospinal fluid antibody testing the practical implication is that the mental health system needs to embrace lumbar puncture as a routine part of practice in high risk groups.

\section{PREDICTORS OF CARER BURDEN IN IMPULSE CONTROL DISORDERS IN PARKINSON'S DISEASE}

Leigh Townsend*, Daniel Johnson, Anthony David, Sally Askey-Jones, Richard Brown, David Okai. Academic Foundation Doctor, University of Oxford; Oxford University Hospitals NHS Foundation Trust

\subsection{6/jnnp-2019-BNPA.26}

Aims/Objectives Impulse control behaviors (ICBs) affect 15\%$35 \%$ of Parkinson's Disease patients. There is evidence of increased carer strain due to these behaviours; however, little is known about clinical variables mediating this effect. This study aims to investigate the factors predictive of carer burden within a cohort of Parkinson's Disease patients with ICBs. Identification of such factors may allow for targeted therapeutic intervention.

Method Data was collected from 45 patients with clinically significant ICBs and their carers including levodopa equivalent daily dosage, assessments of motor and neuropsychiatric symptoms, cognitive function and ICBs. Carer burden was assessed using the Zarit Burden Interview (ZBI). Univariate analyses were performed using Spearman's Rank Correlation Coefficient. A backward model was used to remove variables to create a final multivariate model using ZBI score as the dependent variable.

Results Univariate analysis identified significant correlations between ZBI and total NPI ( $r s=0.50, p<0.0005$ ); four NPI sub-scores: agitation-aggression ( $\mathrm{rs}=0.41, \mathrm{p}=0.005)$, depression-dysphoria $\quad(\mathrm{rs}=0.47, \quad \mathrm{p}=0.001), \quad$ apathy-indifference ( $\mathrm{rs}=0.49, \mathrm{p}=0.001)$ and irritability-lability ( $\mathrm{rs}=0.38, \mathrm{p}=0.03)$; and carer GHQ $(\mathrm{rs}=0.52, \mathrm{p}<0.0005)$. Multivariate linear regression retained total NPI and GHQ scores, collectively predictive of $36.6 \%$ of variance in ZBI.

Conclusions This is the largest study to date, looking at associations between carer burden and ICBs. Our findings indicate low mood, poor motivation, social disinhibition and neuropsychiatric symptom burden to be significant factors in carer burden. We also observe that carers reporting poorer health had increased carer strain. Further work should explore methods of physical and psychosocial support and coping strategies for carers.

\section{MEDICAL STUDENT EDUCATION IN SLEEP AND ITS DISORDERS: HAS IT IMPROVED OVER 20 YEARS?}

${ }^{1}$ Felix May, 'Stephanie Romiszewski, ${ }^{1}$ Ben Norris, ${ }^{2}$ Michelle Miller, ${ }^{3}$ Adam Zeman. ${ }^{1}$ Royal Devon and Exeter NHS Foundation Trust; ${ }^{2}$ Warwick University; ${ }^{3}$ University of Exeter Medical School

10.1136/jnnp-2019-BNPA.27

Objectives/Aims There is growing recognition that sound sleep is a pillar of health, alongside adequate nutrition and exercise. Sleep problems are common and often treatable, improving lives. Twenty years ago, Stores ${ }^{1}$ revealed the paucity of UK medical school-education on sleep disorders, with a median teaching time of $20 \mathrm{~min}$ : we investigate here whether this situation has changed.

Methods A cross-sectional survey of 34 medical degree courses in the UK, adapted from Stores' 1998 questionnaire, including time spent on teaching sleep medicine, sub-topics covered, and forms of assessment. Responses were coded and analysed numerically where possible; free text was analysed thematically. We excluded responses not concerned with general undergraduate education.

Results Twenty-five (74\%) UK medical schools responded to our survey. The time devoted to teaching sleep medicine during undergraduate training was median 1.5 hours, mode$<1$ hour, and mean 3.2 hours (standard deviation $=-2.6$ ).

Only two schools reported a sleep medicine syllabus or dedicated compulsory module (8\%), whilst two had optional student-selected sleep medicine modules (8\%). Sleep medicine was generally described as being subsumed into other areas, primarily respiratory medicine, sometimes ENT, Psychiatry and Neurology; coverage of subtopics mirrored this pattern. Asked if enough time is allotted for teaching on sleep medicine, $50 \%$ said Yes, 38\% No, 13\% were unsure.

Free-text comments made by our respondents had recurring themes: sleep medicine is typically subsumed into teaching by other specialties, consequently course directors are uncertain about the details of provision, obstructive sleep-apnoea is often identified as the key or only relevant sleep disorder, knowledge of sleep disorders is regarded as optional, and there is inertia about the prospect of change. However, a substantial minority of respondents are enthusiastic about making 